\title{
Optimal Configuration of Distribution System considering the Economic Operations of a Microgrid
}

\author{
Hyun-Ho Moon*'Jong-Joo Lee $\cdot$ Sang-Yule Choi $\cdot$ Myong-Chul Shin
}

\begin{abstract}
With the diversification of distribution facilities, existing distributed generation can be subdivided into Microgrids, which are smaller units for application. These Microgrids, subdivided as such and connected to distribution systems, should operate under driving plans that will ensure their economic efficiency and, accordingly, the configuration of those distribution systems that include Microgrids should also be changed.

The perception of the necessity to secure the economic efficiency of distribution systems is gradually increasing and studies intended to assess the economic efficiency of Microgrids and Smartgrids are ongoing.

In this paper, the power generation capacity of an economically operative Microgrid was calculated using the MonteCarlo simulation, which is a method based on the probability theory considering the power generation cost of Microgrids linked with power supply systems and reverse sales costs, etc., and an optimum distribution systems was configured based on the results of these calculation.
\end{abstract}

Key Words : Microgrid, DG, Distribution, Optimal Configuration, Monte Carlo

\section{Introduction}

In modern societies, power consumption is increasing and the types of power consumed in distribution systems are being diversified. With the diversification of distribution systems, distributed

* Main author : Department of Electronic and Electrical Engineering, Sungkyunkwan University (primary author)

Tel : +82-31-467-4746, Fax : +82-31-467-4709

E-mail : hhmoon@daelim.ac.kr

Date of submit : 2009. 11. 3

First assessment : 2009. 11. 6, Second : 2010. 3. 25

Completion of assessment : 2010. 4. 14 generators used in existing systems can be subdivided into smaller units, or microgrids[1]. With the spread of such subdivided power supply systems, existing distribution systems take the form of bilateral as opposed to unilateral flow, and they can be supplied with power from distributed generators rather than from KEPCO to resell unused power to KEPCO.

Accordingly, a microgrid connected to a distribution system should be operated according to an economically efficient operation plan, and the structure of the distribution system should also be changed. 
In order to determine the economic efficiency of distributed generators running connected to a distribution system, the present study calculated the capacity of a microgrid operable economically using the Monte Carlo method, a probability method, in consideration of the generation costs and reverse selling costs of a microgrid operated in connection to the distribution system, and designed a distribution system based on the capacity found.

\section{Economic Operation of DG}

\subsection{Microgrid}

With the introduction of small-size distributed generators and planned control, it is possible to control a power system network that can generate enough power to be operable separately from the main power system network, and part of a power system network with such capacity is called a microgrid [2].

Microgrids include solar power generators, wind turbine generators, fuel cells, micro-turbines, and cogeneration. In order to achieve meaningful results from microgrids, an evaluation of the economic efficiency of microgrids is needed as well as an introduction of criteria for accurate evaluation. In order to evaluate the economic efficiency of a microgrid, the present study calculated the economical generation capacity of a microgrid through a proposed simulation based on the power generation costs of solar power generation, wind turbine generation and fuel cells. The restructuring of distribution systems based on the calculation results are proposed.

\subsection{Monte Carlo Technique}

The Monte Carlo technique, a simulation method, is used to find the probable distribution of a number to be obtained through statistics from repeatable experiments. This method is useful for finding an approximate solution using random numbers for problems that are hard or impossible to solve analytically or numerically using numeric equations or functions[3].

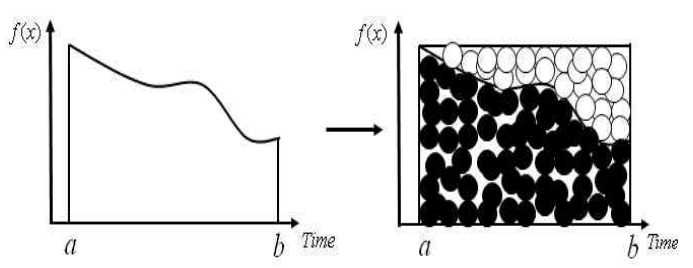

Fig. 1. Monte Carlo integral calculus

An advantage of the Monte Carlo technique is its ease of application. To find the accurate target value, an appropriate algorithm should be developed based on extensive background knowledge including various mathematical formulae. However, the Monte Carlo technique can find a relatively accurate value through simulation without such procedures. On the other hand, one disadvantage of this method is that the result is meaningless if the probability distribution of the input values and the range of mathematical modeling are not correct. Because the distribution of random numbers has a significant effect on the result of analysis, the random number generation function should be formulated properly according to the required range and distribution of random numbers.

\subsection{Simulation Data}

The Monte Carlo simulation is a probability 
method, and the reliability of its results is improved when objective data are used as variables.

In this study, a probability distribution as in Table 1 was calculated based on IEEE RTS daily load data, and the load was determined in proportion to the system to which the simulation was applied. Table 2.3 below shows data for measuring the economic profits of the simulation, which are the electric charge and power generation costs of the Korea Electric Power Corporation and Korea Energy Management Corporation.

Table 1. Probability Distribution by Generation Capability

\begin{tabular}{|c|c|c|c|}
\hline Ratio & $\begin{array}{c}\text { Operating } \\
\text { Time } \\
{[\text { Hour }]}\end{array}$ & $\begin{array}{c}\text { Probability } \\
{[\%]}\end{array}$ & $\begin{array}{c}\text { Accumulative } \\
\text { Probability } \\
{[\%]}\end{array}$ \\
\hline 0.60 & 4 & 0.17 & 0.00 \\
\hline 0.65 & 2 & 0.08 & 0.17 \\
\hline 0.70 & 1 & 0.04 & 0.25 \\
\hline 0.75 & 2 & 0.08 & 0.29 \\
\hline 0.80 & 0 & 0 & 0.38 \\
\hline 0.85 & 1 & 0.04 & 0.38 \\
\hline 0.90 & 1 & 0.04 & 0.42 \\
\hline 0.95 & 7 & 0.29 & 0.46 \\
\hline 1 (Peak) & 6 & 0.25 & 0.75 \\
\hline
\end{tabular}

Table 2. The Power rate of KEPCO in 2008[4]

\begin{tabular}{|c|c|}
\hline Residential [ Won / kWh ] & 55.10 \\
\hline Commercial [ Won / kWh ] & 67.90 \\
\hline Industrial [ Won / kWh ] & 65.80 \\
\hline
\end{tabular}

Table 3. The Power rate of DG

\begin{tabular}{|c|c|}
\hline $\begin{array}{c}\text { Wind Turbine Generator } \\
{[\text { Won / kWh ] }}\end{array}$ & 107.29 \\
\hline $\begin{array}{c}\text { Solar Power Generator } \\
{[\text { Won / kWh ] }}\end{array}$ & 667.38 \\
\hline Fuel Cell [ Won / kWh ] & 282.54 \\
\hline
\end{tabular}

The simulated system to calculate the economical generation capacity and to design the optimal structure based on the capacity is a RBTS 2-bus system, which was provided for testing the reliability of distribution system and the peak load, which is $20[\mathrm{MW}]$.

Table 4. Peak Capacity of Area and Capacity of DG

\begin{tabular}{|c|c|c|}
\hline Areas & $\begin{array}{c}\text { Capacity } \\
{[\mathrm{kW}]}\end{array}$ & $\begin{array}{c}\text { Capacity of DG } \\
{[\mathrm{kW}]}\end{array}$ \\
\hline 1 (Residential) & 5933.8 & 2000 (Wind) \\
\hline 2 (Industrial) & 3500 & 3000 (Wind) \\
\hline 3 (Residential) & 5046.1 & $2000($ PV) \\
\hline 4 (Commercial) & 5520.7 & 3000 (Fuel Cell) \\
\hline
\end{tabular}

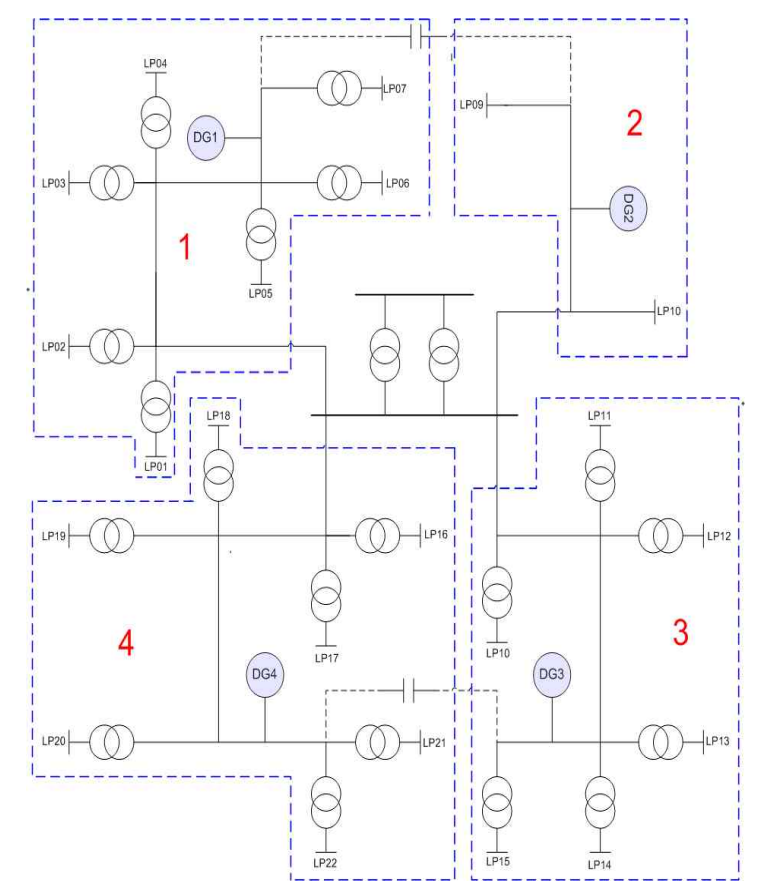

Fig. 2. RBTS 2bus system

In this paper, the distribution system was built by adding four distributed generators to tow existing RBTS buses, and a given load was used as the peak capacity of each load. The entire system was largely divided into four areas, and the existing two RBTS 
buses were modified and applied [5-6]. Each area was again divided as residential area, commercial area, and large-scale industrial area, and the distributed generators applied to each area were wind turbine generation, solar power generation, and fuel cell. The length of distribution line and line load were not considered. The peak load and distributed generation capacity of each area are as in the table below. Fig. 2 is the RBTS 2 bus modifying Table 4 .

\section{Simulation and Optimal Configuration}

\subsection{Monte Carlo Simulation}

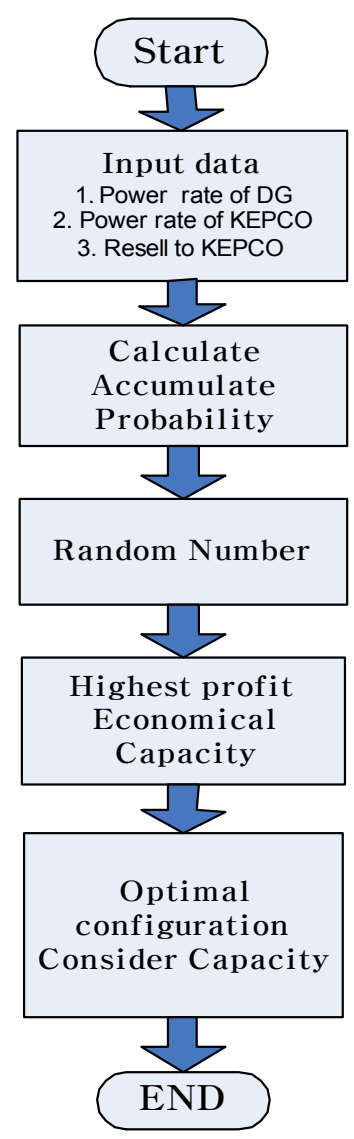

Fig. 3. Monte Carlo Simulation Flow Chart

In order to calculate the generation capacity that maximizes the economic profit in each distributed generator, a Monte Carlo simulation was performed by generating random numbers and calculating probability distribution using a representative Excel spreadsheet and by applying the various simulation data mentioned in the previous chapter. The input data of the simulation were generation cost and sales cost calculated from the data of the Korea Electric Power Corporation and Korea Energy Management Corporation, while probability distribution according to capacity was obtained from daily load. The simulation was repeated five times using 5000 random numbers, and the generation of maximum profit was calculated using the mean values. Fig. 3 is a flowchart of the Monte Carlo simulation in this paper.

\subsection{Economical Generator Capacity of DG}

Figure 4, 5, 6 and 7 below are graphs from the Monte Carlo simulation on the profit of distributed generation according to daily load, showing the capacity of the highest profit for each distributed generator. The profit was highest when the distributed generations were $1700[\mathrm{~kW}]$ (DG1), 2600[kW] (DG2), 1900[kW] (DG3), and 2400[kW] (DG4).

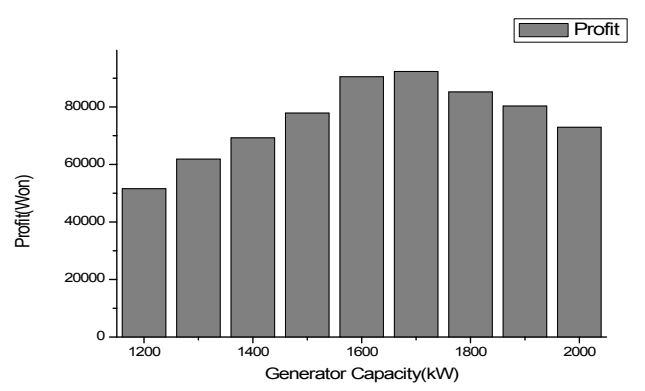

Fig. 4. Profit graph of DG1 (Wind) 


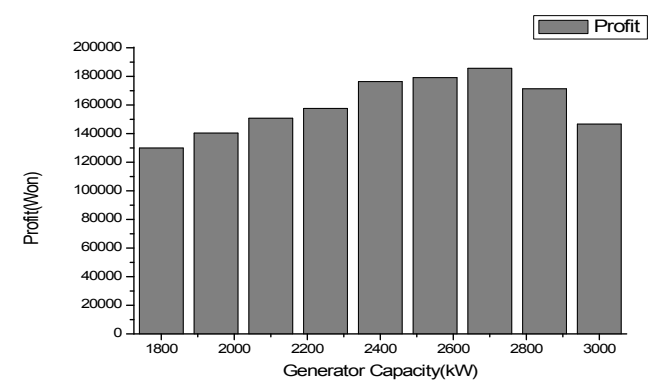

Fig. 5. Profit graph of DG2 (Wind)

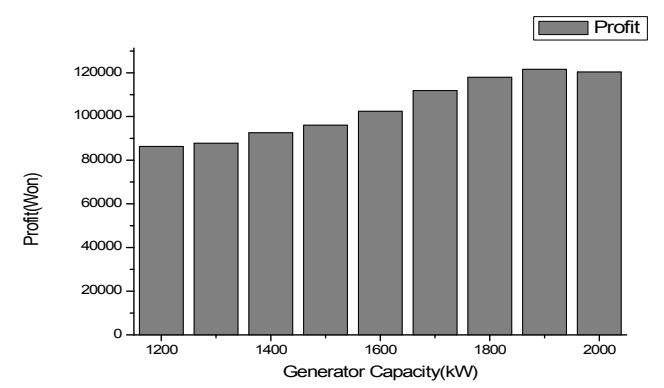

Fig. 6. Profit graph of DG3 (PV)

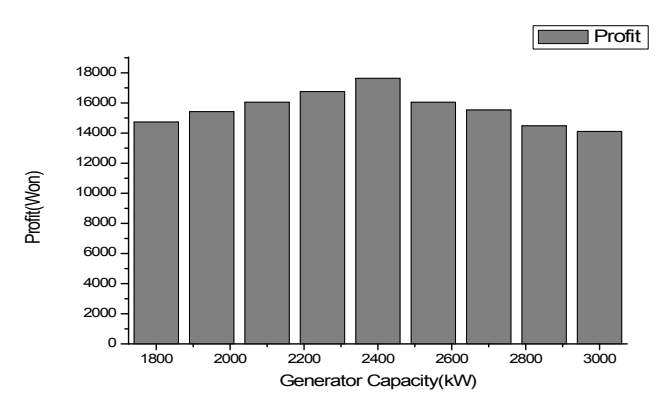

Fig. 7. Profit graph of DG4 (Fuel Cell)

\subsection{Optimal Configuration of Distribution System}

Based on the simulation data and daily load of each distributed generator and through the Monte Carlo simulation, the economic generation capacity of each distributed generator was calculated for the capacity that produces the maximum profit. According to Fig. 8 below, the load of each load point could be met when the modified RBTS 2-bus system was operated at the economic generation capacity. The range that does not cause problems to the entire system is as in Fig. 8.

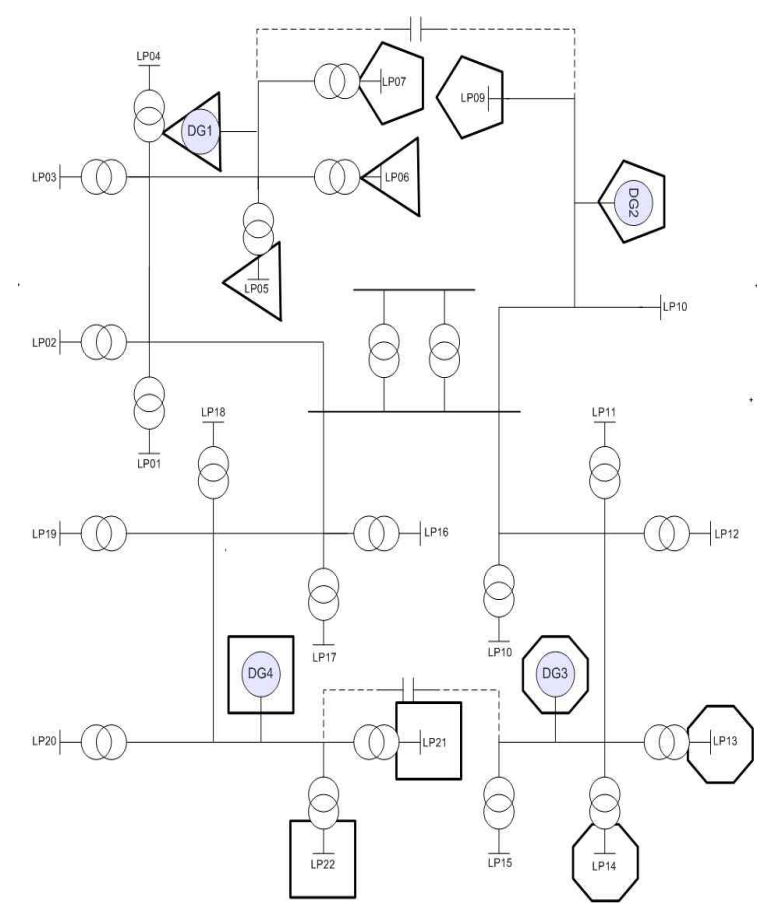

Fig. 8. Optimal configuration of RBTS 2BUS system

\section{Conclusion}

This study suggested a method for calculating generation that produces the maximum profit in distributed generation using a Monte Carlo simulation, which can find approximate values faster than numerical methods, and designed an optimal structure for a distribution system based on the capacity producing the maximum profit. By implementing a Monte Carlo simulation, an economically optimal structure of existing distribution systems with distributed generators and economical distributed generation plans can be suggested. The characteristic data and reliability indexes of distribution lines may be used as basic data of a 
simulation for designing the optimal structure of distribution systems in terms of economic efficiency and quality.

\section{References}

[1] Jong-Bo An Dong-Wook Yoo, "Development of autonomous demand-management type MicroGrid" IEFF Trans. Power Delivery, vol. 3, pp. 549-557, Apr. 1988.

[2] Robert Lasseter, Abbas Akhil, Chris Marnay, "The CERTS MicroGrid Concept" April, 2002.

[3] Jea-Sam Kim, "Monte Carlo Method and Application" Deawoo, 1998.

[4] KPCO Web Site "http://www.kepco.co.kr/" 2008.

[5] R. N. Allan, R. Billinton, I. Sjarief, L. Goel "A Reliability Test System for Educational Purpose- Basic distribution System data and Result", IEIE Transactions on Power System 1991.

[6] E.Ghiani, S. Mocci, Member, F. Pilo "Optimal Reconfiguration of Distribution Networks According to the Microgrid Paradigm" Future Power Systems, Intemational Conference, 2005.

[7] In-Soo Bae, "Optimal Configuration of Virtual Power Plants Considering Reliability Worth and Economic Assessment" Han Yang University, 2007.

[8] Gun-Chang Lee, "New Digital Information Technology" Muyok , 2005

[9] J. Duncan Glover, Mulukutla S. Sarma, "Power System Analysis and Design Third Edition" Brooks/Cole.

[10] E. Ghiani, S. Mocci, F. Pilo, "Optimal Reconfiguration of Distribution Networks According to the Microgrid Paradigm”, Future Power Systems, 2005 International Conf pp 6-12

\section{Biography}

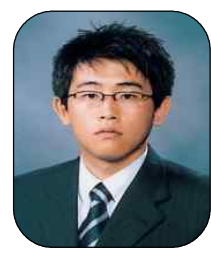

\section{Hyun-Ho Moon}

Mr. Moon received his B.S. degree from the Korea Polytechnic University of Korea in 2004. He received an M.S. degree from Sungkyunkwan University, Korea, in 2006. He is currently a Ph.D candidate at Sungkyunkwan University, Korea. Since 2002, he has been an assistant teacher at Daelim College. His research areas of interests are power system stability and power system control, DG and microgrids.

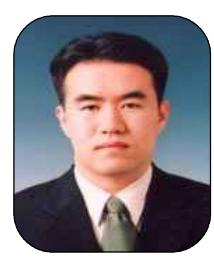

\section{Jong-Joo Lee}

Mr. Lee received his B.S. degree from the University of Suwon, Korea, in 1999. He received an M.S. degree from Sungkyunkwan University, Korea, in 2002. From 2002 to 2004 he was a senior researcher at SATURN Information \& Communications Co., Ltd., Korea, and from 2004 to 2007 he was a senior researcher at Sungkyunkwan University Regional Innovation Center. He received his Ph.D. degree in the Dept. of Information \& Communication Engineering at Sungkyunkwan University, Korea, in 2008.

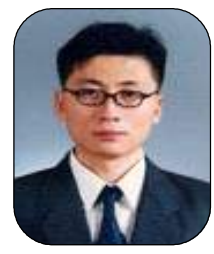

\section{Sang-Yule Choi}

Mr. Choi received his B.S., M.S. and Ph.D. degrees in Electrical Engineering from Sungkyunkwan University, Korea in 1996, 1998 and 2002. From 2002 to 2004 he was a professor at An-Yang University, Korea. $\mathrm{He}$ is currently a professor at Induk Institute University, Korea. His research areas of interests are active database, and the automation algorithm of distribution systems.

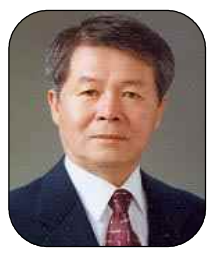

\section{Myong-Chul Shin}

Mr. Shin received a B.S. degree in electrical engineering from Sungkyunkwan University, Seoul, South Korea, in 1970 and M.S. and Ph. D. degrees from Yonsei University, Seoul, South Korea, in 1973 and 1978, respectively.

$\mathrm{He}$ is currently a president of "The Korean Institute of Electrical Engineers (KIEE)" and has been a Professor of Electric and Computer Engineering at Sungkyunkwan University since 1978. He has worked in many areas of computer applications in power systems. His research areas of interests are digital protection of electric power systems, power system stability and power system control. 